\title{
Depositionally-Induced Magnetic Frequency Variations of a Sandstone Facies from the Copper Harbor Conglomerate of the North American, Mid-Continent Rift at Union Bay, Michigan
}

\author{
Elizabeth A. Borucki \\ Department of Geosciences \\ University of Wisconsin-Milwaukee \\ Milwaukee, WI, USA
}

\begin{abstract}
Precambrian sandstones were investigated to determine whether deep-time, fluvial red-beds can maintain magnetic variations related to deposition. Using an easily accessible site on Earth, it is possible to define methods that benefit future studies related to magnetic variations as linked to conditions of formation. The study utilized environmental magnetism and cyclostratigraphic techniques to determine magnetic characteristics and susceptibility variation frequencies. Magnetite and hematite were inferred from laboratory measurements and the magnetic fabric agreed with previous paleocurrent observations, suggesting that susceptibility correlates with deposition. Hematite concentration controlled susceptibility: high-susceptibility corresponded with large grain-sizes and cross-bedding, while low-susceptibility was attributed to small grainsizes and erosional or color boundaries. A statistically-significant, cyclic, susceptibility signal was observed from the stratigraphy, suggesting that susceptibility variations may arise from climatically-induced variations in deposition. The observed frequency also supports the capacity for fluvial red-bed deposits to maintain depositional characteristics original to formational processes.
\end{abstract}

\section{Introduction and Background}

Magnetic properties of rocks and sediments are used to study a wide variety of geologic processes, such as geomagnetic field variations, tectonic reconstructions, and paleoenvironmental variations (Dekkers, 2001). Environmental magnetism concerns itself with high frequency variations such as orientation, concentration, and variability of iron-bearing minerals in relation to fluctuations in the depositional record (Liu et al., 2012). Climatically-driven depositional variations in magnetic properties can sometimes be linked to well-recognized orbital frequencies ranging from tens to hundreds of thousands of years. Such cyclic frequencies have been observed through a field of study known as cyclostratigraphy. Cyclostratigraphy aims to collect high-resolution data over sometimes extended, stratigraphic intervals (Kodama and Hinnov, 2015). In the past, properties such as lithologic or isotopic variations have been used to identify cyclic intervals related to astronomically forced climate changes (e.g., Olsen et al., 1996 and Hays et al., 1976). However, data such as that obtained from the collection of magnetic susceptibility measurements are becoming more widely used in this field. This is due to the relatively non-invasive techniques and high-resolution data that can be acquired from measuring magnetic susceptibility at the outcrop. Iron bearing minerals are reflective of environmental and climatic history since they are sensitive to not only the conditions of their original formation, but also changes in depositional history and post-depositional alteration (Liu et al., 2012), all of which may be influenced by climate.

The author gratefully acknowledges the Wisconsin Space Grant Consortium for the provision of funding through the Dr. Laurel Salton Clark Memorial Research Fellowship. The author also expresses sincere gratitude for the Paleomagnetic Laboratory at the University of Wisconsin-Milwaukee for equipment and assistance as well as the Michigan Department of Natural Resources for graciously permitting work within the Porcupine Wilderness State Park. Finally, the author would like to express the sincerest thanks to Dr. Julie Bowles for her unwavering support and guidance. 
Traditionally, cyclostratigraphic analysis has been best tested on marine sediments as they are mostly representative of continuous deposition. However, Kodama (2016) successfully identified an eccentricity and obliquity signal in the $330 \mathrm{Ma}$ fluvial red beds of the Mauch Chunk formation in Pennsylvania using outcrop based magnetic susceptibility measurements. The Mauch Chunk developed under semi-arid conditions in a sub-aerial to fluvial environment through deltaic progradation (Vrazo et al., 2007). The Mauch Chunk formation shares similarities with the depositional history of the 1.1 Ga Copper Harbor Conglomerate (CHC) which developed under arid conditions and was deposited through alluvial fan deposition that fed into ephemeral bodies of water (Wolff and Huber, 1973; Elmore, 1984).

This study focuses on a small section of the Porcupine Wilderness State Park, Michigan, that hosts a sandstone facies of the CHC. The measurements and samples for this research were selected and obtained from a small section representative of fine- to medium-grained sandstone that featured a variety of sedimentary bed-forms. The overall goal is to determine if it is possible to isolate a cyclic signal linked to climate or deposition in the Precambrian rock record, using magnetic susceptibility measured from the outcrop. Susceptibility is influenced by mineralogy, grain size, or the concentration of iron-bearing minerals as it measures the total response of all material to an induced magnetic field. In addition to the measurement of outcrop magnetic susceptibility, the magnetic mineral abundance, arrangement, composition, and grain size were also characterized in the lab. Previous studies at this site observed detrital magnetite, martite, and specularite as well as authigenic pigmentary hematite clay rims and coarse hematite crystal grains (Elmore and van der Voo, 1982). The detrital components were found to maintain a depositional remanence and the authigenic components carried a secondary, post-depositional remanence (Elmore and van der Voo, 1982). The hematite fraction was observed to be significantly greater relative to magnetite although the exact nature and source of the hematite remanence is somewhat unclear, due to its presence as variable forms. Fortunately, the work presented here focused on magnetic susceptibility and not magnetic remanence. The significant abundance of hematite in the $\mathrm{CHC}$ deposits will be the dominant factor behind any outcropbased magnetic susceptibility measurements.

Several arguments that support susceptibility's ability to be reflective of a depositional signal, even if the remanence does not, include: that regardless of magnetic grain size, all magnetic material contributes to susceptibility including detrital and small magnetic grains that won't necessarily contribute to magnetic remanence (Elmore and van der Voo, 1982); therefore, despite some alteration of magnetic minerals occurring post-deposition, the abundance of magnetic minerals matters more than when it altered, as the altered minerals continue to act as a placeholder for the original detrital minerals. The variations in magnetic mineral abundance are presumably related to depositional variations. This is because an altered product of the detrital mineral would maintain any depositional cycles associated with the original mineral even after the transformation. Similarly, any form of hematite that resulted from the replacement of a depositional mineral should retain the depositional signal. Therefore, the characterization of magnetic properties enable the assessment and degree to which magnetic mineralogy may be linked to depositional variations at this locality. 


\section{Field and Laboratory Methodology}

Twenty-nine block samples were collected on average every 2.67 stratigraphic meters at minimum intervals of $0.09 \mathrm{~m}$ to maximum intervals of $26.8 \mathrm{~m}$ from the outcrop in accordance with the permit granted by the Michigan Department of Natural Resources for use of state land. Block samples were supplemental for outcrop-based, magnetic susceptibility measurements. In the lab, susceptibility was measured as a function of temperature $(\chi(T))$ using a CS4 furnace attachment for the Agico MFK1 Kappabridge under air atmosphere. Curie temperatures were estimated by taking the peak in the first derivative of the $\chi(\mathrm{T})$ data after applying a furnace correction and smoothing the data with a running-mean filter (Fabian et al., 2013). Roomtemperature bulk susceptibility $\left(\chi_{\mathrm{B}}\right)$, and anisotropy of magnetic susceptibility (AMS) were measured using the MFK1 Kappabridge susceptibility bridge. Specimens acquired detailed isothermal remanent magnetization (IRM) through a 20-step-acquisition process up to $1 \mathrm{~T}$, followed by a single back-field IRM at 0.3 T using an ASC Model IM10-30 impulse magnetizer. All remanence measurements were conducted on a $2 \mathrm{G}$ Enterprises 755SRMS Superconducting Rock Magnetometer housed inside the shielded room at the University of Wisconsin-Milwaukee Paleomagnetic Laboratory. The backfield enabled calculation of the S-ratio and Hard-IRM. The S-ratio is defined as -IRM-300mT/IRM1T where IRM-300mT is the backfield $0.3 \mathrm{~T}$ IRM acquired following application of the forward $1 \mathrm{~T}$ field (Liu et al., 2012). When an S-ratio is near +1 the data is representative of a greater proportion of soft minerals within the sample, such as magnetite, while a value near -1 would be indicative of a greater contribution from harder minerals such as hematite. The Hard-IRM $=0.5 \times($ IRM1T + IRM-300mT) and the Soft-IRM = IRM1T - Hard-IRM were also calculated. Respectively, these represent the portion of the IRM carried by high-coercivity and low-coercivity magnetic minerals. Detailed IRM acquisition data was decomposed in terms of coercivity spectra. This technique was implemented using the first derivative of the IRM vs. applied field data, following methodology developed by Robertson and France (1994), Stockhausen (1998), Kruiver et al. (2001), Heslop et al. (2002), Egli (2003), Heslop and Dillion (2007), and Heslop (2015), modified by Maxbauer et al. (2016). Detailed IRM un-mixing are reported in terms of the peak coercivity of each skew-normal distribution and the dispersion about that peak. Typically, a larger dispersion parameter indicates a wider range of magnetic grain sizes. To calculate the fractional contribution of each component to the IRM, the integrated area under each component is calculated and extrapolates any non-saturated components to higher coercivities.

To conduct outcrop susceptibility measurements, the KT-10 v2 S/C Magnetic Susceptibility Meter was used. The susceptibility meter applies an alternating field and derives susceptibility from the response frequency difference between the outcrop and the free-air. The outcrop-based magnetic susceptibility measurements were collected stratigraphically and were obtained to achieve high-resolution information due to the necessarily sparse physical sample collection permitted at this outcrop location. Accurate spectral analysis of the stratigraphic variations in susceptibility requires frequent measurements that are evenly spaced and accurately located. Outcrop susceptibility readings were taken up-section, stratigraphically, over a total of $56.4 \mathrm{~m}$. Measurements were taken a minimum of every $0.01 \mathrm{~m}$ and a maximum distance of $1.5 \mathrm{~m}$ apart, with an average spaced interval of $0.47 \mathrm{~m}$. A spectral analysis of the susceptibility data series was conducted following the protocol outlined by Kodama and Hinnov (2015). First, the data series was resampled at the average sampling rate of $0.47 \mathrm{~m}$ using a simple linear interpolation 
(Kodama et al., 2010). A linear trend was removed from the data series using Matlab ${ }^{\mathrm{TM}}$ polynomial curve fitting and evaluation. The multitaper spectral analysis of the data utilized a

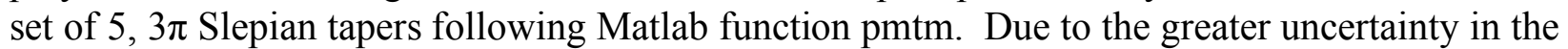
positioning of the data, the $3 \pi$ Slepian taper was used rather than the $2 \pi$ Slepian taper that was recommended by Kodama et al. (2010) to assist with greater smoothing of the data. The greater smoothing diminishes the spectral resolution but increases confidence in the spectral peaks. Significance of the spectral peaks was estimated by performing a Monte Carlo simulation $(\mathrm{N}=1000)$ of a red noise spectrum, and resulted in $80 \%, 85 \%, 90 \%$, and $99 \%$ confidence limits. Elevation of data spectral peaks above the red noise background level suggests these frequency peaks are not noise. The red noise spectrum is thought to best represent climate and geological time series (Kodama and Hinnov, 2015). Red noise gives greater power to lower frequency rather than high frequency noise, and unlike white noise spectrums that are completely independent of frequency behavior, red noise behavior retains a "memory" (Kodama and Hinnov, 2015). This memory means that prior behavior influences future behavior (Kodama and Hinnov, 2015).

To examine variability at length scales $<0.5 \mathrm{~m}$, two separate grid sections were established to measure susceptibility variations within bed-forms. Grid 1 was mapped out on a flat outcrop surface with a grid system measuring $90 \times 40 \mathrm{~cm}$. The grid was sectioned off into $5 \times 5 \mathrm{~cm}$ squares, which created eight columns vertically down the grid and eighteen rows horizontally across the grid. Susceptibility was measured within each $5 \times 5 \mathrm{~cm}$ square. Grid 2 was also mapped adjacent to Grid 1 on the same outcrop surface. This grid was mapped out in an irregular rectangular shape to avoid rough sections of the outcrop. The horizontal length of the grid was $310 \mathrm{~cm}$, and the vertical extent varied from $170 \mathrm{~cm}$ on the left to $230 \mathrm{~cm}$ on the right. Vertically from top-to-bottom, susceptibility measurements were taken every $5 \mathrm{~cm}$.

\section{Results and Observations}

Temperature dependent susceptibility measurements consistently exhibited two magnetic components, one high and one low coercivity. Heating curve Curie temperatures $\left(\mathrm{T}_{\mathrm{C}}\right)$ ranged from 395 to $637^{\circ} \mathrm{C}$ but consistently exhibited $\mathrm{T}_{\mathrm{C}}$ near $550-572^{\circ} \mathrm{C}$, suggestive of low-Ti titanomagnetite (magnetite $\mathrm{T}_{\mathrm{C}}=580^{\circ} \mathrm{C}$ ). Approximately half of the specimens demonstrated an additional $\mathrm{T}_{\mathrm{C}}$ in the range of $600-680^{\circ} \mathrm{C}$, suggestive of compositions ranging from titanohematite to hematite (hematite $\mathrm{T}_{\mathrm{C}}=675^{\circ} \mathrm{C}$ ). 
IRM acquisition was unable to saturate the specimens even in a 1T field, reflective of a high- coercivity component such as hematite. Results revealed two primary magnetic components for each individual specimen: a soft component that had an average peak coercivity of 51.24 $\mathrm{mT}$ and a narrow dispersion parameter of $1.84 \mathrm{mT}$. The hard component demonstrated significantly higher coercivity, with an average peak coercivity of $630.55 \mathrm{mT}$ and an average dispersion parameter of 2.40 $\mathrm{mT}$. The average contributions of the soft and hard component were $9.37 \% \pm$ $1.09 \%$ and $90.63 \% \pm 1.09 \%$, respectively. The S-ratio, hard- and soft-IRM parameters seem to provide similar information on abundance and relative proportions of hard and soft minerals as did the detailed IRM unmixing results indicated above. The Sratio average was $-0.33 \pm-0.08$ and was found to vary from -0.69 to -0.15 . These consistently negative S-ratio values support a significant presence of hard minerals, such as hematite, that
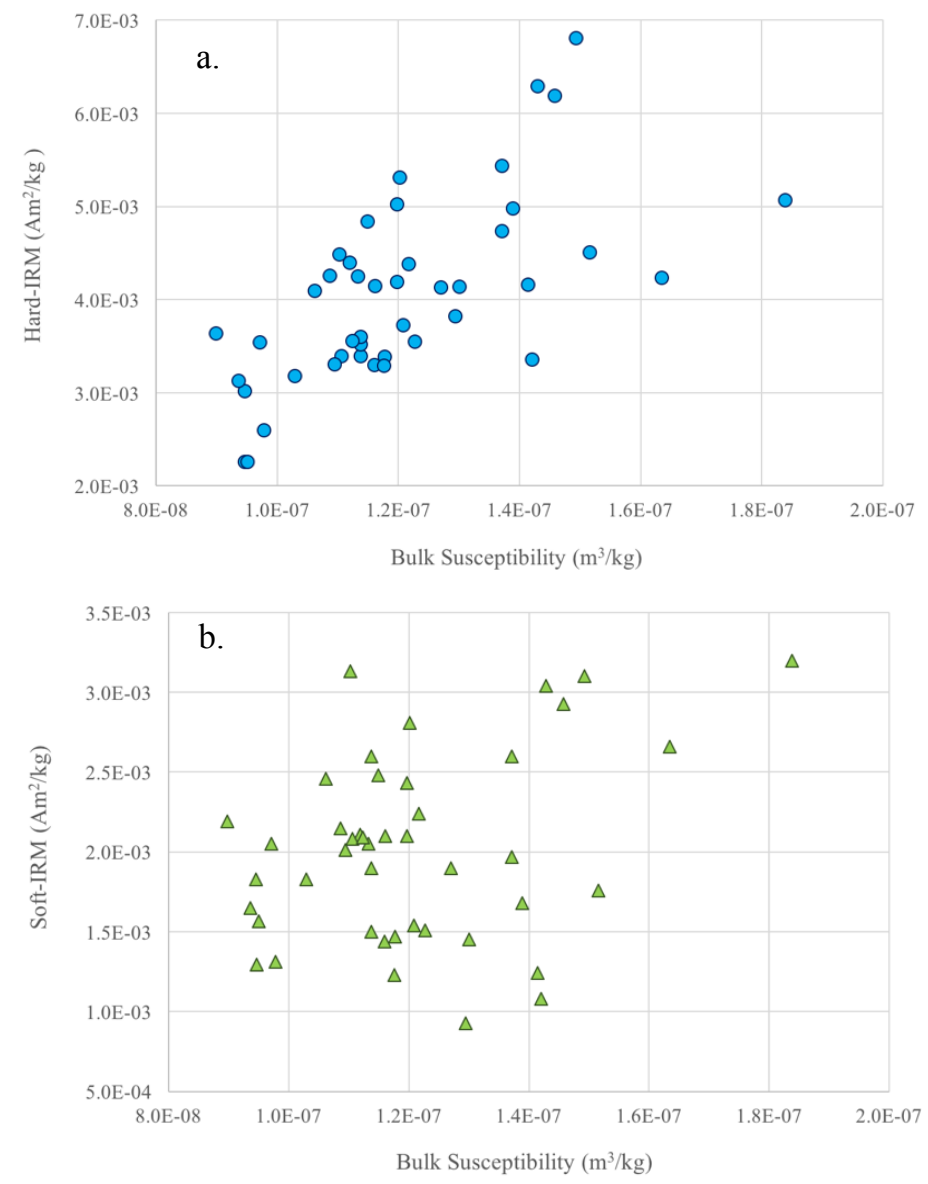

Fig 1. (a.) The hard-IRM demonstrates a positive correlation with susceptibility, the (b.) soft-IRM does not.

was inferred from the inability of the samples to reach full saturation in a $1 \mathrm{~T}$ field and as was identified through temperature dependent susceptibility. The S-ratio correlated positively with the extrapolated contribution of the soft component meaning that as the S-ratio became less negative there was a greater contribution from the softer minerals. The hard-IRM average (4.13 $\left.\times 10^{-3} \mathrm{Am}^{2} / \mathrm{kg} \pm 0.001\right)$ was greater than that of the soft-IRM average $\left(2.10 \times 10^{-3} \mathrm{Am}^{2} / \mathrm{kg} \pm\right.$ 0.001 ) again suggestive that the hematite contribution is greater than that of magnetite. Although the correlations are imperfect, calculated IRM's generally follow the expected trends. Both the hard-IRM, when compared with the contribution from the hard component, and the soft-IRM, when compared with the contribution from the soft component, demonstrated positive trends. Therefore, both hard- and soft-IRM correspond with abundance of hard or soft minerals, respectively. The hard- and soft-IRM were also compared with overall bulk susceptibility, while the hard-IRM demonstrated a positive, linear trend (Fig 1a), the soft-IRM did not display a clear correlation (Fig. 1b). Overall, susceptibility seems to correlate with the magnetic remanence associated with hematite. This correlation is important because it would suggest that hematite and not magnetite control the outcrop-based susceptibility frequency variations observed in the spectral analysis below. 

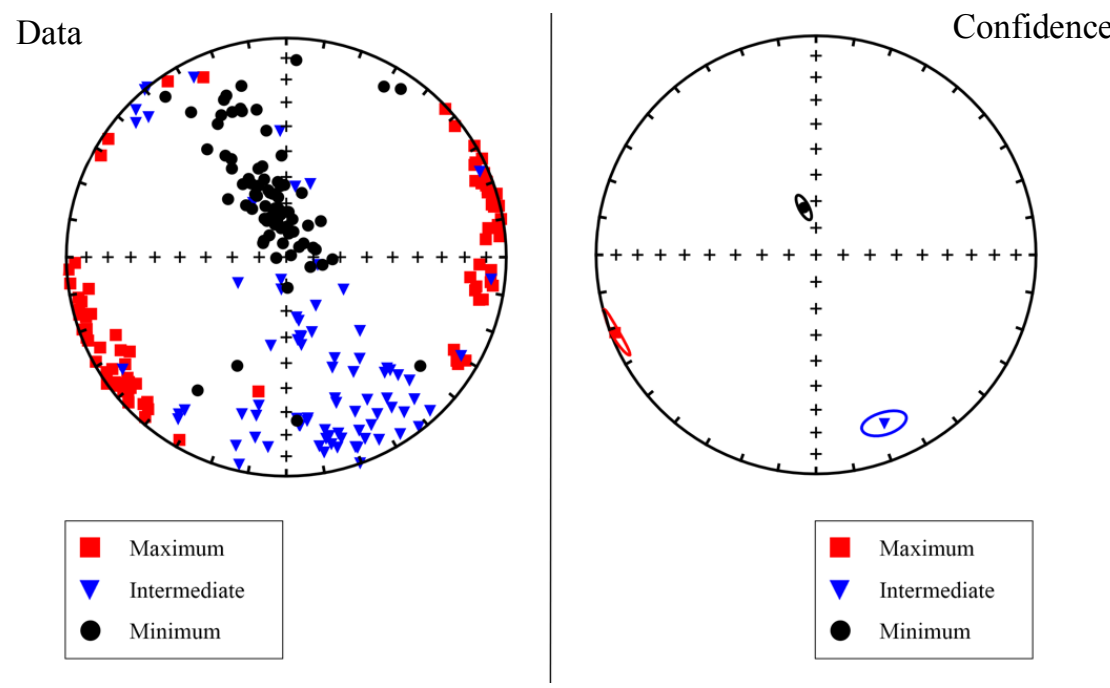

Fig 2. The data are plotted in the lower hemisphere. The smearing of the minimum axes across the center of the plot is suggestive of deposition through turbulent flow. The maximum axes are oriented perpendicular to flow, in support of paleoflow directed towards the NW. The confidence was determined using the parametric bootstrap method (Tauxe, 2016). The means and alpha-95 of the data sets are shown using confidence ellipses.

Because the $\mathrm{CHC}$ bedding has a regional tilt of approximately $21^{\circ}$ towards the N-NE, the resulting AMS data were corrected for tilt. The AMS shape was determined to be primarily prolate and AMS data, at the site level, place the minimum axes as clustered and smeared across the vertical stereoplot axis trending mostly within the NW quadrant. The maximum axes are mostly clustered within the SW and NE quadrants of the stereoplot within the horizontal plane (Fig. 2). The smearing of the minimum axes suggests that the mode of transportation may have been through turbulent flow and the paleocurrent was likely moving perpendicular to the maximum axes (Tauxe, 2016). Such an interpretation would suggest that the paleocurrent that deposited these magnetic grains was moving either towards the NW or SE, with preference towards the NW based on the clustering of the minimum axes in the NW quadrant. Previous paleocurrent measurements near Union Bay that were based on pebble imbrication, by Wolf and Huber (1973), determined a NE to NW paleocurrent direction, consistent with these AMS results. This outcome supports that depositional orientation is maintained in this succession through susceptibility.

The magnetic susceptibility was found to increase up section. Application of a simple 5-point running mean highlighted periodicity in the data, and examination of the spectral analysis identified cyclical frequencies from the susceptibility measurements (Fig. 3). One peak with a significant frequency every $\sim 2.08 \mathrm{~m}$ was statistically distinct from the background robust red noise estimation with $>99 \%$ confidence. Additionally, two broader peaks of lower confidence were present in the

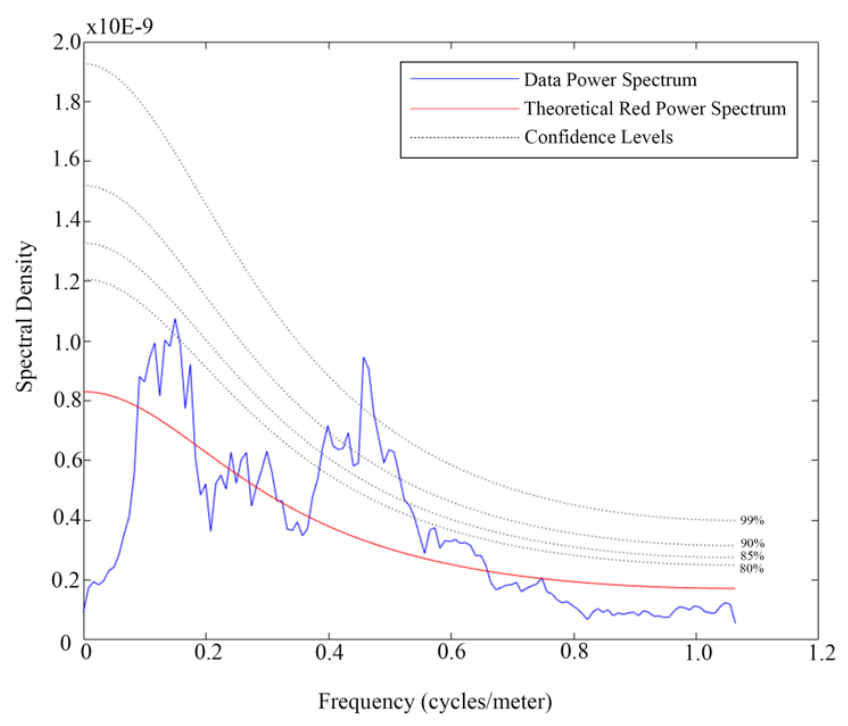

Fig 3. The data power spectrum (blue curve) results from the spectral analysis of outcrop-based magnetic susceptibility measurements collected in the field. The robust red noise (red curve) was estimated using the Monte-Carlo simulation. Peaks above the red noise curve are distinct from background noise. Peaks above the confidence limits (black dashed lines) represent significant peaks. 
spectral output. The broadest peak ranged between frequency significances approximately every $1.79 \mathrm{~m}-2.50 \mathrm{~m}$, and represents peaks statistically distinct with $>90 \%$ confidence. A peak with $>80 \%$ confidence represents an approximate frequency of significance every $5.88 \mathrm{~m}$.

The purpose of both small, outcrop susceptibility grids was to demonstrate the amount of magnetic variability at a spatial scale smaller than the $\sim 0.5 \mathrm{~m}$ spacing which was used for the entire stratigraphic section. The first grid contains a section of well sorted, fine- to medium-grained, crossbedded, sandstone (Fig. 4a). High susceptibility was measured near the base of the grid and near the top of the grid (Fig. 4b.). The high susceptibility near the base of the grid is associated with the approximate location of the steeply dipping foresets with greater grain size variation, while the high susceptibility near the top of the grid is associated with a thick band of lower medium-grained sandstone. Meanwhile the section of low susceptibility bounded between these sections of high susceptibility is representative of finegrained sandstone (Fig. 4a \& b). Grid 2 outlines a section of cross-beds of wellsorted, fine- to lower medium-grained, cross-bedded sandstone (Fig. 5a). The
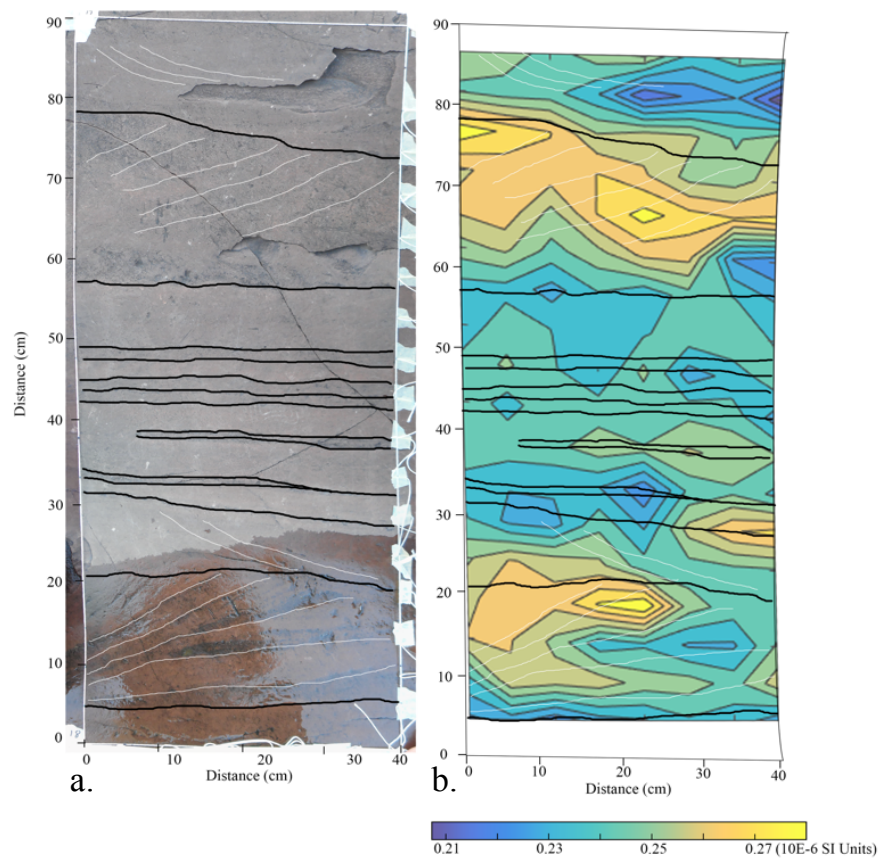

Fig 4. Grid 1 Outcrop-based magnetic susceptibility variations. (a.) Depicts bedding contacts with black lines and bedding features with white lines. (b.) Demonstrates relatively high (warm colors) and low (cool colors) susceptibilities measured on the grid surface, bedding contacts and features from Fig. 4(a) for reference. magnetic susceptibility of Grid 2 was not measured as comprehensively as was done for Grid 1, rather three vertical transects were measured to estimate variability within this section of the outcrop (Fig. 5b). The vertical transects show similar susceptibility trends, suggesting roughly consistent along-bedding magnetic variability (Fig. 5b). This is suggestive of steady deposition longitudinally, over a scale of $310 \mathrm{~cm}$. In general, the susceptibility lows seem to correspond to either erosional, bedding, or color change boundaries while susceptibility highs exist within these bounds (Fig. 5a).

\section{Discussion and Significance of Results}

Since the susceptibility signal is dominated by hematite, the cyclical variations we observe may be unrelated to depositional processes or conditions considering that Elmore and van der Voo (1982) observed both detrital and authigenic hematite at Union Bay. A secondary signal produced from the formation of completely new magnetic minerals could obscure the magnetic variations related to the original depositional signal. There are several arguments to be made, however, that the susceptibility reflects a depositional signal, even if the remanence does not. First, Elmore and van der Voo (1982) maintained that the hematite remanence may in fact be a 

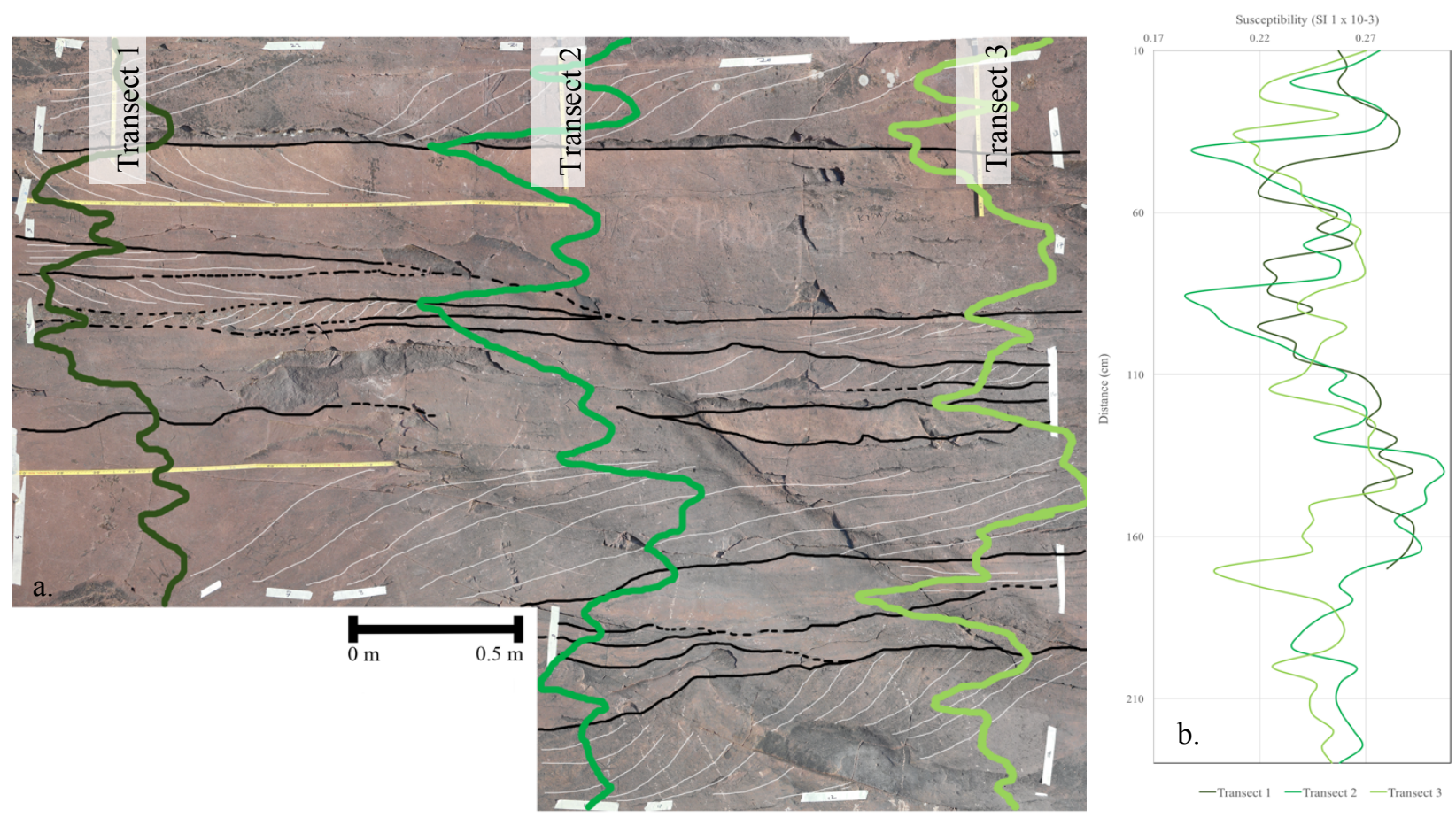

Fig 5. Grid 2 Outcrop-based magnetic susceptibility variations. (a.) Erosional/bedding boundaries are annotated with black lines, bedding features depicted with white lines, and massive sections left un-marked. Green lines of various shades reference relatively high and low susceptibly that was measured along each vertical transect. These lines correspond with the susceptibility measurements from the (b.) graph to the right of the annotated, outcrop image.

vector average of pre- and post-depositional hematite. Second, some hematite, as determined by Elmore and van der Voo (1982), is detrital and superparamagnetic in size, which is too small a grain size to contribute to magnetic remanence; however, it will contribute to susceptibility measurements. Third, although some martitization is believed to have occurred post-deposition, the abundance of martite matters more than when it altered, and variations in abundance are presumably related to depositional variations. This is because the martitization is an altered product of the detrital magnetite so any depositional cycles associated with the martite variation should remain, even after the transformation from magnetite to hematite. The martite more or less acts as a placeholder for the original magnetite. Similarly, any form of hematite that resulted from the replacement of a depositional mineral should retain the depositional signal. Only if entirely new hematite precipitates as the result of the transportation of ions into the matrix will the post-depositional hematite be of greater concern since it won't be reflective of a detrital signal. It is possible though that if new minerals are produced, their concentration may still reflect some aspect of the original depositional materials, such as porosity, which means that the original depositional signal could still be maintained. Fourth, the paleocurrent direction determined from the AMS fabric is consistent with the direction determined from bedding features. The anisotropy as related to magnetic susceptibility clearly reflects a depositional orientation. However, one could imagine a secondary, authigenic hematite forming around depositionally aligned minerals, resulting in a distribution anisotropy (Hargraves et al., 1991).

To examine in more detail the underlying physical variations that may be linked to susceptibility variations, the high-resolution susceptibility grids may be useful. Grid 1 demonstrated susceptibility variations associated with shifts in grain size. Lower medium grains 
(approximately 0.25 millimeters), the coarser of the grain sizes present within this grid, and the foresets at the base of the grid, which represent a wider range of grain size variation than the rest of the grid, were both associated with higher susceptibility measurements. These areas of high susceptibility may have been influenced by an influx of different source materials during deposition that had greater abundance of magnetic minerals. Another explanation could be that the foresets at the base of the grid and the areas with larger grain sizes may have yielded greater interstitial space or secondary voids, possibly a result of well sorted grains, meaning low grainto-grain contacts. Greater voids would have provided more space thus, higher porosity, within which authigenic, box-work hematite could grow and latch on to the matrix minerals that cemented the sandstone together during diagenesis. Low susceptibility was observed within the middle of the grid between the top and bottom bounds of higher susceptibility, and was associated with fine-grained sandstone. The fine-grained sandstone likely represents less interstitial space meaning less secondary hematite could develop and thus presents with lower susceptibility measurements. This line of thought would suggest that susceptibility variability is representative of a secondary trait of deposition, such as porosity rather than a changing influx of depositional magnetic materials.

High and low susceptibility measurements of Grid 2 seem to associate with bed-forms, color changes, or erosional boundaries. Most of the lowest susceptibility measurements correlated well with erosional boundaries or color changes while high susceptibility existed between these lows and were associated with large cross beds. The susceptibility highs within the cross beds were likely influenced by an influx of material that either brought greater abundance of magnetic materials or provided greater void space for accumulation of authigenic hematite. Meanwhile erosional boundaries potentially represent a shift to finer grain sizes of lower porosity as represented by low susceptibility measurements. Additionally, low susceptibility was observed at a color change boundary that seemed to be expressed laterally across the grid along the same color contact. The red bed color of the $\mathrm{CHC}$ has often been attributed to the fine-grained pigmentary hematite associated with either pre- or post-depositional clay rims. At this color boundary, the sandstone's pigment shifted from a deep red to a light, bleached red which may indicate a changing concentration of pigmentary hematite, from high to low. The color shift may be related to less dehydration of iron-bearing silicates within the dune feature that formed in an arid environment, likely influenced by occasional overland floods. Since there was a lack of vegetation during the Precambrian, every rainfall event could potentially have produced overland flooding on an alluvial fan surface such as the setting for this locality. If such rainfall events were related to climate, they could potentially influence increased or decreased dehydration of the iron-bearing silicates present at the site. Based on the observations made at the finer timescales captured by both grids, high and low susceptibilities seem to be closely related to bedding features and characteristics. Therefore, these results suggest that the presence of hematite is primarily controlled by the physical characteristics of the deposit and the observed cyclicity is a result related to changes in the depositional regime, not of a post-depositional environmental change that resulted in an increase of hematite.

If chronology was better established for these facies or if there was an estimated accumulation rate, it would be possible to determine a true periodicity for these successive, susceptibility measurements, allowing for a more complete spectral analysis. Unfortunately, the CHC formation occurs entirely within a normal magnetic polarity interval (Ojakangas et al., 2001). 
Without polarity boundaries or other clear indications of accumulation rates for this stratigraphic succession, it is unfeasible to conduct a full cyclostratigraphic analysis. Nevertheless, by accepting that this susceptibility data plausibly reflects climatically-influenced variations, it is possible to begin to consider what the cycles might represent. The susceptibility spectral data output can be compared with known Milankovitch cycles to assess the feasibility that the variations are orbitally-forced. The frequencies determined from the spectral output indicated a spectral peak of $>99 \%$ confidence approximately every $2.08 \mathrm{~m}$. This frequency would represent accumulation rates for short eccentricity $(100 \mathrm{kyr})$ at $0.021 \mathrm{~mm} / \mathrm{yr}$, long eccentricity (413 kyr) at $0.005 \mathrm{~mm} / \mathrm{yr}$, precession (26 kyr) at $0.080 \mathrm{~mm} / \mathrm{yr}$, and obliquity $(41 \mathrm{kyr})$ at $0.051 \mathrm{~mm} / \mathrm{yr}$. Peaks of lower confidence were also compared with these known Milankovitch intervals, and these calculated accumulation rates were comparable to what was calculated for the $>99 \%$ confidence peak. Although sedimentation rates are highly variable and basin specific, an example of the sedimentation rate averaged over the entire lake basin from the East African rift, of Lake Tanganyika, was approximated at $0.477 \mathrm{~mm} / \mathrm{yr}$ (Cohen et al., 1993). Another example of sedimentation rates includes sediments of the Precambrian Belt Basin of the Northwestern United States that fed into a sea at an estimated sedimentation rate of approximately 0.051 $\mathrm{mm} / \mathrm{yr}$ (Harrison, 1972). Although the rates from this study (assuming orbital forcing) don't seem completely unreasonable when compared with the proposed Precambrian Belt Basin sedimentation rates, due to the uncertainty of the depositional timescale of this section it is impulsive to consider these as true sedimentation rates. Additionally, the CHC sediment accumulation was in a lacustrine setting, not a marine environment. So, it is possible that these measured frequencies are representative of shorter period climatic fluctuations of the Precambrian. Modern analogs for shorter climatic fluctuations that affect temperature, rainfall, and degree of humidity or aridity include the El Niño Southern Oscillation (ENSO) or the Pacific Decadal Oscillation. ENSO occurs over brief intervals of approximately nine to twelve months at a time every three to seven years, on average (Malone et al., 2014). The Pacific Decadal Oscillation often persists for extended periods from 15-20 years or 50-70 years at a time (Mantua and Hare, 2002).

This environmental magnetic study of the CHC of Union Bay support the hypothesis that outcrop susceptibility measurements are indeed reflective of depositional conditions in fluvial red-beds. Furthermore, cyclicity was distinguished from these $1.1 \mathrm{Ga}$ deposits using magnetic susceptibility. The repeatability of the observed frequencies suggests a potential relationship to climate though we cannot determine the scale. Ultimately, these observations support the ability and importance of red beds of fluvial origin to maintain depositional characteristics that provide insight into deep time even if the deposits are dominated by secondary, magnetic minerals. 


\section{References}

Cohen, A. S., Soreghan, M. J., \& Scholz, C. A. (1993). Estimating the Age of Formation of Lakes: An Example from Lake Tanganyika, East African Rift System. Geology, 21, 511-514.

Dekkers, M. J. (2001). Rockmagnetism and Paleomagnetism. In Encyclopedia of Life Support Systems. (Vol. II, 1-3) Utrecht, Netherlands: Geophysics and Geochemistry.

Elmore, R. D., \& van der Voo, R. (1982). Origin of Hematite and its Associated Remanence in the Copper Harbor Conglomerate (Keweenawan), Upper Michigan. Journal of Geophysical Research, 87(B13), 918-928.

Elmore, R. D. (1984). The Copper Harbor Conglomerate: A Late Precambrian Fining-Upward Alluvial Fan Sequence in Northern Michigan. Geological Society of America Bulletin, 95, 610-617.

Egli, R. (2003). Analysis of the Field Dependence of Remanent Magnetization Curves. Journal of Geophysical Research, 108(B2), 1-25.

Fabian, K., Shcherbakov, V.P., and McEnroe, S. A. (2013). Measuring the Curie Temperature. Geochemistry, Geophysics, Geosystems, 14, 947-961.

Hargraves, R. B., Johnson, D., and Chan, C. Y. (1991). Distribution Anisotropy: The Cause of AMS in Igneous Rocks? Geophysical Research Letters, 18, 2193-2196.

Harrison, J. E. (1972). Precambrian Belt Basin of Northwestern United States: Its Geometry, Sedimentation, and Copper Occurrences. Geological Society of America Bulletin, 83, 1215-1240.

Hays, J. D., Imbrie, J., \& Shackleton, N. J. (1976). Variations in the Earth's Orbit: Pacemaker of the Ice Ages. Science, 194(4270), 1121-1132.

Heslop, D. (2015). Numerical Strategies for Magnetic Mineral Un-Mixing. Earth-Science Review, 150, $256-284$.

Heslop, D., \& Dillion, M. (2007). Unmixing Magnetic Remanence Curves without a Prior Knowledge. Geophysics Journal International, 170, 556-566.

Heslop, D., Dekkers, M. J., Kruiver, P. P., \& van Oorschot, I. H. (2002). Analysis of Isothermal Remanent Magnetization Acquisition Curves Using the Expectation-Maximization Algorithm. Geophysical Journal International, 148(1), 58-64.

Kodama, K.P. (2016). Rock Magnetic Cyclostratigraphy of the Mississippian Mauch Chunk fm. Pottsville, Pennsylvania, Abstract GP51D-05, presented at 2016 AGU Fall Meeting, San Francisco CA, 12-16, Dec.

Kodama, K. P., \& Hinnov, L. A. (2015). Rock Magnetic Cyclostratigraphy. Chichster West Sussex, England: John Wiley \& Sons.

Kodama, K. P., Anastasio, D. J., Newton, M. L., Pares, J. M., \& Hinnov, L. A. (2010). High-Resolution Rock Magnetic Cyclostratigraphy in an Eocene Flysch, Spanish Pyrenees. Geochemistry, Geophysics, Geosystems, 11, 1-22.

Kruiver, P. P., Dekkers, M. J., \& Heslop, D. (2001). Quantification of Magnetic Coercivity Components by the Analysis of Acquisition Curves of Isothermal Remanent Magnetization. Earth and Planetary Science Letters, 189, 269276.

Liu, Q., Roberts, P. A., Larrasoana, J. C., Banerjee, S. K., Guyodo, Y., Tauxe, L., \& Oldfield, F. (2012). Environmental Magnetism: Principles and Applications. Reviews of Geophysics, 50 (RG4002), 1-50.

Mantua, N. J. \& Hare R. S. (2002). The Pacific Decadal Oscillation. Journal of Oceanography, 58, 35-44.

Malone, S. L., Staudhammer, C. L., Oberbauer, S. F., Olivas, P., Ryan, M. G., Schedlbauer, J. L., Loescher, H. W., Starr, G. (2014). El Niño Southern Oscillation (ENSO) Enhances $\mathrm{CO}_{2}$ Exchange Rates in Freshwater Marsh

Ecosystems in the Florida Everglades. PLOS ONE 9(12), 1-30.

Maxbauer, D. P., Feinberg, J. M., \& Fox, D. L. (2016). MAX UnMix: A Web Application for Un-mixing Magnetic Coercivity Distributions. Computers and Geosciences, 95, 140-145.

Ojakangas, R. W., Morey, G. B., \& Green, J. C. (2001). The Mesoproterozoic Midcontinent Rift System Lake Superior Region, USA. Sedimentary Geology (141-142), 421-442.

Olsen, P. E., Kent, D. V., Cornet, B., Witte, W. K., \& Schlische, R. W. (1996). High-Resolution Stratigraphy of the Newark

Rift Basin (early Mesozoic, Eastern North American). Geological Society of America Bulletin, 108(1), 40-77.

Robertson, D. J., \& France, D. E. (1994). Discrimination of Remanence-Carrying Minerals in Mixtures Using Isothermal Remanent Magnetization Acquisition Curves. Physics of the Earth Planetary Interiors, (82), 223-234.

Stockhausen, H. (1998). Some New Aspects for the Modelling of Isothermal Remanent Magnetization Acquisition Curves by Cumulative log Gaussian Functions. Geophysical Reserarch Letters (25), 2217-2220.

Vrazo, M. B., Benton, M. J., Daeschler, E. B. (2007). Tetrapod tracks from the Mauch Chunk Formation (middle to upper Mississippian) of Pennsylvania, U.S.A. The Academy of Natural Sciences of Philadelphia, 156(1), 199-209.

Wolff, R. G., \& Huber, N. K. (1973). The Copper Harbor Conglomerate (Middle Keweenawan) on Isle Royale, Michigan, and its Regional Implications. Geological Survey Professional Paper 754-B, United States Department of the Interior. Washington D.C. 